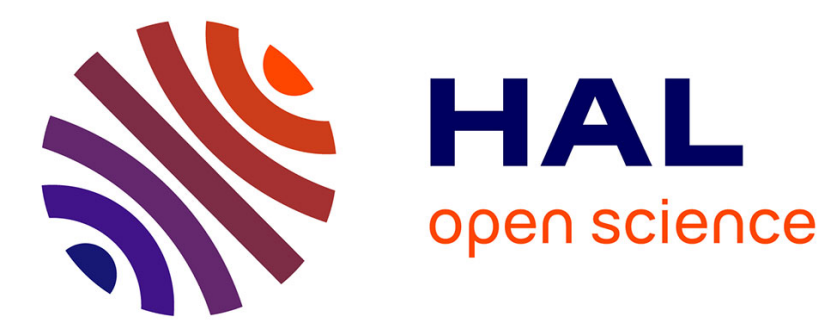

\title{
La proposition introduite par comme quoi
}

Florence Lefeuvre

\section{To cite this version:}

Florence Lefeuvre. La proposition introduite par comme quoi. Lingvisticae Investigationes, 2003, XVI, 2 (XVI, 2), pp.259-283. halshs-00138070

\section{HAL Id: halshs-00138070 https://shs.hal.science/halshs-00138070}

Submitted on 23 Mar 2007

HAL is a multi-disciplinary open access archive for the deposit and dissemination of scientific research documents, whether they are published or not. The documents may come from teaching and research institutions in France or abroad, or from public or private research centers.
L'archive ouverte pluridisciplinaire HAL, est destinée au dépôt et à la diffusion de documents scientifiques de niveau recherche, publiés ou non, émanant des établissements d'enseignement et de recherche français ou étrangers, des laboratoires publics ou privés. 


\title{
LA PROPOSITION INTRODUITE PAR COMME QUOI
}

\author{
FLORENCE LEFEUVRE \\ Université de Brest - Lattice (UMR CNRS 8094)
}

L'objet de cet article est de proposer une synthèse approfondie et réajustée de nos travaux de recherches sur la proposition introduite par comme quoi. Celle-ci est généralement perçue comme relevant du registre familier ou infra-standard. Elle peut se rencontrer dans trois types de constructions. Premièrement, la proposition en comme quoi peut assumer une fonction par rapport au verbe principal, généralement la fonction de complément d'objet direct :

(1) a. La cousine a écrit comme quoi c'était toi pour les pièces de cinq francs dans la cagnotte. (Queffelec, Les Noces barbares)

Deuxièmement, elle est accolée à un syntagme nominal :

(2) a. On pourra étudier posément son foie, son cerveau, son coeur, ses reins, ses poumons, ses muscles, son sang. L'information précipitée comme quoi ce pâtre congelé, et peut-être épileptique, aurait été castré selon un rite initiatique du néolithique [...] se révèle, pour finir, sans fondement. (Sollers, Le Secret)

Troisièmement, elle peut survenir dans un emploi détaché de la phrase précédente. Elle est alors située en début d'énoncé, souvent après une ponctuation forte :

(3) a. C'est un fait que l'épanouissement de ce journal, dans sa continuité a été exceptionnel. Pour cela il aura fallu 40 ans. Comme quoi un journal ne se fait pas en un jour, ni en un tour de table. Il aura fallu le travail obstiné d'une très petite équipe rédactionnelle [...]. (Le Monde diplomatique, janvier 1995)

A la lecture des exemples (1a) et (2a), il n'est pas évident de savoir à quel type de proposition subordonnée celle en comme quoi correspond. En outre, dans l'exemple (3a), l'énoncé en comme quoi, qui apparaît après une ponctuation forte, s'écarte de l'emploi habituel d'une subordonnée. Les grilles d'études sur les subordonnées sont aussi nombreuses que complexes. Wilmet (1997: 541) propose une classification qui s'appuie sur la « nature de l'enchâsseur » et sur "la fonction de l'enchâssée ». Sont distinguées ainsi les sous-phrases adjectivales, pronominales et conjonctionnelles. Riegel et al. (1994) propose de différencier les relatives (dans lesquelles il range les relatives adjectives et les relatives substantives), les complétives (où se côtoient les complétives proprement dites en "que", les constructions infinitives, les constructions interrogatives et exclamatives) et les circonstancielles. Nous pourrions multiplier les exemples. Aucune ne nous a paru satisfaisante pour savoir où "ranger" la proposition en comme quoi. Pour cette étude, nous utiliserons le cadre d'étude mis en place dans Le Goffic 1993 (43) : il a le mérite d'opérer une distinction claire entre les relatives avec antécédent et les relatives sans antécédent, et il met à part les interrogatives indirectes, ce qui est crucial pour l'analyse de la proposition en comme quoi. Selon cet auteur, quatre types de subordonnées «saturent un cadre logique», la percontative (interrogative indirecte), l'intégrative (relative sans antécédent et circonstancielle en $q u$ - ou si), la relative et la complétive. La proposition en comme quoi connaît une tension entre son processus propre et 
son air de ressemblance avec l'une ou l'autre de ces subordonnées. Tout d'abord, nous mettrons en évidence les distinctions entre la proposition en comme quoi et les propositions avec lesquelles elle est généralement rapprochée. Ensuite, nous montrerons en quoi elle se rapproche et se distingue d'une percontative. Enfin, nous examinerons ses principaux emplois.

\section{Distinction avec la relative, la complétive et l'intégrative}

La proposition en comme quoi se différencie des relatives, des complétives et des intégratives en quoi.

\subsection{Différences avec la relative}

Lorsque comme quoi suit un syntagme nominal, en (2a) ou en :

(4) a. Je vous fais un petit papier comme quoi vous êtes venue (énoncé entendu, 15 décembre 2001).

(5) On a des plaintes comme quoi vous n'accordez pas les $4 \%$ de réduction. (Fallait y penser, France 2, 4 décembre 2001)

(6) Il faut que je retrouve la preuve comme quoi j'ai acheté mon ticket. (énoncé entendu, vendredi 14 juin 2002)

(7) [...] et puis vous savez quoi? L'histoire comme quoi il rejouerait au base-ball, elles n'ont pas un instant pensé que leur frère y croyait. (Le Nouvel Observateur, 18-24 juillet 2002)

(8) a. Il a jamais reçu les messages comme quoi mon père était tombé (énoncé entendu, 3 décembre 2001)

l'hypothèse peut-être la plus immédiate consiste à lire cette proposition comme une relative. A première vue, elle ressemble en effet à la structure introduite par le groupe préposition + quoi :

(9) a. [...] elle avait entretenu pour lui la fiction selon quoi Rink et elle s'insupportaient (Labro, Des Bateaux dans la nuit)

(10) a. Il s'agissait d'abattre des arbres pour le petit pont, car on manquait de chevalets, de poutrelles et de madriers sur quoi on poserait le tablier de planches (Rambaud, La Bataille)

Elle est juxtaposée pareillement à un syntagme nominal; surtout, elle comporte le terme quoi qui peut faire penser au pronom relatif quoi. J. Damourette et É. Pichon (1911-1940 (tome 7) : 362) opèrent une telle analyse.

A l'inverse, nous pensons que quoi, en (2a) et dans les énoncés de (4a) à (8a), ne compose pas un relatif. Plusieurs arguments peuvent être avancés en faveur de cette dernière hypothèse. Premièrement, il est aisé de remarquer que, dans les exemples (9a) et (10a), le pronom relatif lequel peut facilement se substituer à quoi :

(9) b. elle avait entretenu pour lui la fiction selon laquelle Rink et elle s'insupportaient

(10) b. on manquait de chevalets, de poutrelles et de madriers sur lesquels on poserait le tablier de planches

Les noms qui précédent la proposition en comme quoi peuvent être utilisés avec le même degré de précision mais la substitution de quoi par lequel s'avère impossible : 
(2) b. *L'information précipitée comme laquelle ce pâtre congelé, et peut-être épileptique, aurait été castré selon un rite initiatique du néolithique [...] se révèle, pour finir, sans fondement

Ce constat peut être effectué pour tous les exemples où comme quoi suit un nom, que celui-ci soit précédé d'un article défini ou indéfini :

(4) b. *Je vous fais un petit papier comme lequel vous êtes venue

Il suffit de substituer, à comme, la préposition selon pour que le changement devienne acceptable :

(2) c. L'information précipitée selon quoi / laquelle ce pâtre congelé, et peutêtre épileptique, aurait été castré selon un rite initiatique du néolithique [...] se révèle, pour finir, sans fondement

Deuxièmement, dans les phrases qui comportent le groupe préposition $+q u o i$, par exemple en (9a), il est possible d'obtenir deux phrases telles que :

(9) c. Elle avait entretenu pour lui cette fiction. Selon cette fiction, Rink et elle s'insupportaient

contrairement à la proposition en comme quoi, ainsi en (8a) :

(8) b. Il a jamais reçu ces messages. *Comme ces messages, mon père était tombé

Ces faits montrent que la proposition en comme quoi ne constitue pas une relative : quoi ne reprend pas un antécédent, à l'opposé du quoi en (9a) et (10a); quoi en (2a) avec comme quoi et quoi en (2c) avec selon quoi ne correspondent pas au même référent (cf. troisième partie). Le quoi de (2c) fonctionne en revanche comme lequel en (2c) parce que tous deux renvoient au même antécédent ${ }^{1}$.

\subsection{Différences avec la complétive}

Selon une deuxième hypothèse, la proposition en comme quoi s'apparenterait à une complétive. Quelquefois la substitution s'avère possible, notamment lorsque comme quoi apparaît avec des verbes déclaratifs, en (1a) et :

(11) a. François risqua le tout pour le tout. Il raconta au vieux comme quoi Guillaume Gourvennec désirait le voir mourir le plus vite possible, et, d'abord, ne plus le garder chez lui. En donnant de l'or à Guillaume, on arrangerait l'affaire (Queffelec, Un Recteur de l'île de Sein).

(12) Mais moi je me souvenais comme quoi elle a travaillé sur la traduction (énoncé entendu, le 3 juillet 2002)

(13) Ce que je peux faire, c'est mettre comme quoi elle a eu la mention TB (énoncé entendu, le 2 juillet 2002)

(14) Tu montres comme quoi tu fais un effort toi aussi (énoncé entendu, 6 janvier 2002)

\footnotetext{
${ }^{1} \mathrm{Il}$ n'est pas de notre propos ici de montrer la différence entre quoi relatif et lequel relatif (cf. Lefeuvre à paraître c).
} 
Les énoncés suivants peuvent être obtenus sans aucune difficulté :

(1) b. La cousine a écrit que c'était toi pour les pièces de cinq francs dans la cagnotte

(11) b. il raconta au vieux que Guillaume Gourvennec désirait le voir mourir le plus vite possible

On obtiendrait le même résultat avec les énoncés (12), (13) et (14). Il est parfois possible de remplacer, par une complétive, la proposition en comme quoi lorsqu'elle suit un syntagme nominal abstrait, introduit par un déterminant défini et qui ait un rapport avec le dire, comme en $(15 \mathrm{a})$ :

(15) a. J'ai eu la confirmation comme quoi c'était OK. Tout ça par oral. (énoncé entendu, 4 mars 2002)

(15) b. J'ai eu la confirmation que c'était $O K$.

En outre, comme quoi se caractérise, dans ces énoncés, par un sens ténu ${ }^{2}$, qui rappelle celui du complétif que. Enfin, après comme quoi et le complétif que, se trouve une structure de phrase complète. Ce rapprochement a été plusieurs fois opéré, par exemple par W. Von Wartburg et P. Zumthor (1989: 84) qui parlent d'une " conjonction de subordination complétive », par K. Sandfeld (1977 : 71) qui évoque « la forme forte de la conjonction que » ou encore par G. Le Bidois et R. Le Bidois (1971) qui comparent comme quoi à « un simple outil de subordination $»$.

Sans contester que le rapprochement puisse être effectué dans ces exemples, il nous semble intéressant de montrer de quelle façon la proposition en comme quoi se distingue de la complétive. Pour ce qui est de la première structure, lorsque la proposition en comme quoi revêt une fonction par rapport à un verbe, il existe des verbes qui peuvent accepter la structure en comme quoi mais pas une complétive :

(16) a. J'ai vu chez nous des mères pleurer, on les avait dénoncées à la police comme quoi elles avaient un môme dans le métier qu'elles faisaient et elles mouraient de peur (Ajar, La Vie devant soi)

(16) b. *on les avait dénoncées à la police qu'elles avaient un môme dans le métier

En ce qui concerne la construction syntagme nominal + comme quoi, les exemples ne peuvent pas être paraphrasés par une complétive s'ils comportent un nom concret :

(4) a. Je vous fais un petit papier comme quoi vous êtes venue (pigiste du Monde, 15 décembre 2001)

(4) c. *Je vous fais un petit papier que vous êtes venue

ou s'ils sont précédés d'un déterminant indéfini :

(17) a. Il a fait tout un discours comme quoi il a pas supporté son médecin (énoncé entendu, 3 décembre 2001)

(17) b. *Il a fait tout un discours qu'il a pas supporté son médecin.

Même avec les noms abstraits qui sont introduits par un déterminant défini, la correspondance ne s'établit pas forcément :

${ }^{2} \mathrm{Si}$ ténu soit-il, le sens de comme quoi existe : cf. notre deuxième partie. 
(2) d. ?L'information précipitée que ce pâtre congelé, et peut-être épileptique, aurait été castré selon un rite initiatique du néolithique [...] se révèle, pour finir, sans fondement.

\subsection{Différences avec l'intégrative 3 en quoi (relative sans antécédent)}

Selon une troisième hypothèse, la proposition en comme quoi qui apparaît après une ponctuation forte :

(3) a. C'est un fait que l'épanouissement de ce journal, dans sa continuité a été exceptionnel. Pour cela il aura fallu 40 ans. Comme quoi un journal ne se fait pas en un jour, ni en un tour de table. Il aura fallu le travail obstiné d'une très petite équipe rédactionnelle [...]. (Le Monde diplomatique, janvier 1995)

serait de la même famille que les énoncés introduits par l'ensemble préposition $+q u o i$ :

(18) a. Ce samedi-là, en fin d'après-midi, le Premier ministre m'a téléphoné pour m'annoncer qu'il remaniait le gouvernement et mettait fin à mes fonctions. Ce fut un entretien très bref. J'avoue avoir éprouvé un choc. Sincèrement, je ne m'attendais pas à cette décision. J'ai eu besoin de m'enfermer, seule, dans mon bureau pour accuser le coup. Après quoi, comme si de rien n'était, je suis allée retrouver les poètes du Printemps de la Poésie et j'ai passé une soirée avec eux dans un petit restaurant. Et puis le lendemain, dimanche 26 mars, j'ai participé, sans rien dire de ce qui m'arrivait, à l'émission de Paul Amar. (Le Nouvel Observateur, mars 2001).

L'expression de «relatif de liaison» revient assez souvent pour désigner ce type d'occurrences ${ }^{4}$. En (18a), quoi renvoie à la prédication précédente, ce qui pourrait se paraphraser par :

(18) b. Après m'être enfermée seule dans mon bureau pour accuser le coup, comme si de rien n'était, je suis allée retrouver les poètes du Printemps de la Poésie.

Mais aucun antécédent nominal (/ pronominal) n'est construit, contrairement aux énoncés en préposition + quoi qui s'appuient sur le pronom ce :

(19) [...] il présente bel et bien Val Waxman comme un cinéaste doué et talentueux, parfaitement à même, sans doute, de diriger ses films " à l'oreille ”. Ce en quoi Waxman n'est pas le premier, souvenons-nous par exemple de Marcel Pagnol [...]. (Le Nouvel Observateur, 30 mai-5 juin 2002)

En (18a), à cause de cette absence d'antécédent nominal, nous n'analysons pas, comme un relatif, le terme $q u o i^{5}$. Celui-ci oscille entre un statut de subordonnant intégratif et, en raison, entre autres, de la ponctuation forte qui le sépare nettement de l'énoncé précédent, un statut de

\footnotetext{
3 Pour ce terme, cf. P. Le Goffic $1993: 45$.

${ }^{4}$ H-D. Béchade 1994 : 298 ; H. Mirault $1997: 118$.

${ }^{5} \mathrm{Cf}$. F. Lefeuvre 2001.
} 
simple indéfini indépendant ( $\mathrm{cf}$. F. Lefeuvre à paraître b). Ce type d'énoncé pourrait être considéré comme parataxique, c'est-à-dire comme un intermédiaire entre l'indépendance syntaxique et l'hypotaxe.

Examinons à présent si, dans la structure en comme quoi en début d'énoncé, quoi renvoie à l'énoncé précédent de la même façon qu'en (18a). C'est une analyse qui est régulièrement proposée et comme quoi peut être alors perçu comme un relatif de liaison ${ }^{6}$. Mais contrairement à (18a) qui accepte une paraphrase avec après que, il n'est pas possible de paraphraser (3a) ni par le subordonnant comme :

(3) b. ?Comme c'est un fait que pour l'épanouissement de ce journal, il aura fallu 40 ans, un journal ne se fait pas en un jour, ni en un tour de table

ni par un autre subordonnant :

(3) c. ?Étant donné que c'est un fait que pour l'épanouissement de ce journal, il aura fallu 40 ans, un journal ne se fait pas en un jour, ni en un tour de table

Ces paraphrases explicitent un lien causal. Elles semblent étranges lorsque, comme dans cet exemple, le décrochage entre un énoncé à caractère particulier ( $c^{\prime} e s t$ un fait que pour l'épanouissement de ce journal, il aura fallu 40 ans) et un énoncé à caractère généralisant (un journal ne se fait pas en un jour, ni en un tour de table) est trop important. Les paraphrases les plus proches de l'énoncé (3a) ajoutent un verbe ou un nom qui ont un rapport avec le dire :

(3) d. Comme la phrase précédente le montre, un journal ne se fait pas en un jour

(3) e. Ce qui vient d'être dit montre qu'un journal ne se fait pas en un jour

(3) f. Ce qui vient d'être dit est la preuve qu'un journal ne se fait pas en un jour $^{7}$

Elles s'éloignent de la simple reprise de la proposition précédente tel que c'est le cas en (18a). Cette impossibilité pour la structure en comme quoi de se paraphraser simplement à l'aide d'un subordonnant révèle qu'elle se distingue de celle introduite par le groupe préposition + quoi. Il nous semble plus pertinent, nous le verrons dans notre deuxième partie, d'analyser ce type d'énoncés en comme quoi par rapport aux deux autres constructions possibles, telles (1a) et (2a).

Les éléments que nous venons de considérer montrent que la proposition en comme quoi diffère de la relative, de la complétive et de l'intégrative. Reste à examiner le cas de la percontative.

\section{Ressemblances et différences avec les interrogatives}

Après avoir mis en évidence l'origine interrogative de la proposition en comme quoi, nous montrerons en quoi elle se distingue des percontatives (interrogatives indirectes).

\subsection{Une origine d'interrogative}

${ }^{6}$ Cf. par exemple, H. Mirault $1997: 119$.

7 Pour ce type de paraphrases, cf. H. Mirault 1997 (119); W. Von Wartburg et P. Zumthor 1989 (79) ; Le Goffic 1993 (373). L'indicision semble de mise puisque W. Von Wartburg et P. Zumthor (1989 : 129) évoquent également le sématisme de la cause ou de la conséquence et que Le Goffic (1993) donne une glose du type (3c). 
L'étude diachronique éclaire l'emploi du groupe comme quoi $i^{8}$. Celui-ci apparaît au $17^{\mathrm{e}}$ siècle, en concurrence de comment et de comme, dans une interrogative directe :

(20) Comme quoy les rescompensoit Marc Antoine? (Scudéry, L'Apologie du théâtre, 1639)

et dans une percontative :

(21) Je ne sçay comme quoy vous pourrez vous sauver (Guérin de Bouscal, Dom Quixote de la Manche, 1639)

Cet emploi est présenté comme nouveau dans plusieurs ouvrages du XVIIe, par exemple dans Les Remarques de la langue française de Vaugelas (1647) et dans la grammaire de Chiflet (1659). A cette époque, les emplois de comme et de comment sont encore en concurrence?. Mais l'utilisation de comme interrogatif était alors controversée ${ }^{10}$. Selon nous, une des solutions fut d'accoler, à l'adverbe comme, le pronom interrogatif quoi. L'ajout de ce dernier a permis de ne plus voir en comme un interrogatif mais un intégratif qui s'articule sur le terme quoi et, en (21), la proposition vous pourrez vous sauver. Ainsi la proposition en comme quoi provient d'une interrogative.

$\mathrm{Au}$ cours du temps, le sémantisme de comme quoi a évolué. Au $17 \mathrm{e}$ siècle, dans les exemples mentionnés ci-dessus, il pouvait se comparer à la valeur de comment. De nos jours, en revanche, sa valeur s'est modifiée, sauf dans de rares exemples qui sonnent étrangement à l'oreille du lecteur moderne :

(22) Pour fermer le ban, Marie-France [...] s'est suicidée. Couic. Voilà comme quoi elle s'était conclue, lamentable, l'épopée des hamsters maudits à la baraque (Bayon, Le Lycéen, 1987)

Le sémantisme de comme quoi correspond à une valeur énonciative, comparable à celle qui apparaît dans ce type d'énoncé :

(23) Comme je vous l'ai dit, à cette vue, j'étais tombé sans connaissance (Luzel, Nouvelles veillées bretonnes).

L'adverbe comme porte sur l'analogie entre je vous l'ai dit et sur à cette vue, j'étais tombé sans connaissance. Il renvoie aux conditions d'énonciation. L'exemple (23) peut se gloser par :

"dans les mêmes conditions d'énonciation que je vous ai dit qu'à cette vue, j'étais tombé sans connaissance, de cette façon, dans ces mêmes conditions d'énonciation, à cette vue, j'étais tombé sans connaissance"11

Il indique l'analogie des conditions d'énonciation entre le fait et le discours sur le fait. De même, la proposition en comme quoi ne possède pas la même signification qu'au $17^{\mathrm{e}}$ siècle mais revêt un sens énonciatif : comme quoi signale les conditions d'énonciation du dire et présente une appréciation de la force assertive. Ce qui ressort en effet des deux premiers

\footnotetext{
${ }^{8} \mathrm{Cf}$. F. Lefeuvre à paraître $\mathrm{a}$.

${ }^{9}$ Cf. N. Fournier $1998: 125$.

${ }^{10}$ Cf. C. Vaugelas (de) $1647: 194$

${ }^{11}$ Cf. P. Le Goffic 1991.
} 
emplois, tels en (1a) et (2a), c'est que le type de verbes ou de noms par rapport auquel comme quoi s'articule possède un sens en rapport avec le dire. La structure en comme quoi ne peut pas constituer le complément essentiel direct (COD) d'un verbe qui ne comporte pas ce sème :

(24) *Cette révélation fait comme quoi tu monteras en grade

Elle ne peut pas non plus suivre des syntagmes nominaux qui posséderaient une autre signification :

\section{(25) *La chaise comme quoi}

Dans les exemples qui comportent comme quoi, du type (1a), puisque comme s'emploie avec le même type de verbes qu'en (23), nous pensons qu'il constitue un adverbe intégratif qui s'articule sur le terme quoi et sur P2, par exemple, en (1a), sur c'était toi pour les pièces de cinq francs dans la cagnotte. Le groupe comme quoi pourrait être analysé comme une subordonnée elliptique, qui se gloserait par : "comme il est dit quoi" et, en (1a), "comme elle a écrit quoi / telle ou telle chose" 12 , ce qui peut se gloser de la façon suivante :

"dans les mêmes conditions d'énonciation que la cousine a écrit que c'était toi pour les pièces de cinq francs dans la cagnotte, dans ces mêmes conditions d'énonciation, c'était toi pour les pièces de cinq francs dans la cagnotte"

Quoi compose un pronom indéfini percontatif qui balaie toutes les valeurs possibles sans sélection :

"la cousine a écrit que c'était toi pour les pièces de cinq francs dans la cagnotte ou elle a écrit quelque chose d'approchant".

Nous analyserons, selon un schéma comparable, les deux autres constructions du type (2a) et (3a). Dans le cas de (1a), la source énonciative est précisée. Pour la structure syntagme nominal + comme quoi, c'est le type de discours (2a) ou bien le support (papier en 4a) qui est indiqué, ce qui donne pour (2a) :

"dans les mêmes conditions d'énonciation que l'information précipitée dit que ce pâtre congelé $[\ldots]$ aurait été castré selon un rite initiatique du néolithique ou quelque chose d'approchant, dans ces mêmes conditions d'énonciation, ce pâtre congelé [...] aurait été castré selon un rite initiatique du néolithique "

Lorsque comme quoi se trouve en début d'énoncé, ainsi en (3a), l'origine du dire reste indéterminée, ce qui incite à utiliser le passif. :

"dans les mêmes conditions d'énonciation qu' il est dit qu'un journal ne se fait pas en un jour, ni en un tour de table ou quelque chose d'approchant, dans ces mêmes conditions d'énonciation, un journal ne se fait pas en un jour, ni en un tour de table".

Il existe cependant un rapport entre cette structure en comme quoi et les énoncés précédents, ce qui permet d'avancer que la source énonciative se trouve dans les phrases précédentes :

12 Cf. F. Lefeuvre 2002. 
"comme les phrases précédentes le disent / montrent, un journal ne se fait pas en un jour, ni en un tour de table"

Selon cette interprétation, la proposition en comme quoi provient d'une percontative, avec pour comme, un rôle d'intégratif et pour quoi, un rôle d'interrogatif. Mais à présent elle s'est éloignée des caractéristiques propres à une percontative et elle a acquis un statut particulier.

\subsection{Une proposition à part}

La proposition en comme quoi se distingue des percontatives en ce que l'interrogative directe correspondante n'est pas usitée :

(1) c. *Comme quoi était-ce toi pour les pièces de cinq francs dans la cagnotte?

Comme quoi n'apparaît dans une interrogative directe qu'avec le sens de la comparaison :

(26) Beau comme quoi? (Tournier, Le Coq de Bruyère)

En outre, la proposition en comme quoi s'éloigne des emplois habituels des percontatives. Les verbes qui peuvent s'utiliser avec comme quoi ne sont pas toujours ceux qui s'emploient avec une percontative. Ainsi en est-il du verbe dénoncer dans l'exemple déjà mentionné :

(16) a. J'ai vu chez nous des mères pleurer, on les avait dénoncées à la police comme quoi elles avaient un môme dans le métier qu'elles faisaient et elles mouraient de peur (Ajar, La Vie devant soi)

(16) c. *on les avait dénoncées à la police comment

A l'inverse, des verbes utilisés ordinairement avec une percontative ne peuvent pas régir la proposition en comme quoi:

(27) Il demande comment tu t'appelles

(28) *Il demande comme quoi

Contrairement aux percontatives qui s'articulent le plus souvent par rapport à un verbe, la structure en comme quoi liée à un verbe est peu fréquente de nos jours. Cela dit, elle assure alors, généralement, des fonctions similaires, notamment celle de COD. Plus rarement elle est susceptible d'assumer une autre fonction, par exemple celle de COI (exemple 12) ou celle de sujet :

(29) L'évolution du complot contre la sûreté intérieure de l'état est ainsi nettement indiquée et comme quoi il devait aboutir à une entente avec l'ennemi en vue d'un résultat qui pourrait être obtenu qu'en favorisant ses entreprises. (Le Procès du Maréchal Pétain)

Elle peut s'articuler sur le verbe être et endosser la fonction d'attribut. Il en est ainsi de l'exemple suivant où un déménageur présente un papier à signer et déclare :

(30) C'est comme quoi je l'ai monté [le canapé] chez vous. (énoncé entendu, 25 juillet 2002)

Le sens en rapport avec le dire est donné par le $c^{\prime}$ qui désigne le papier en question. 
Plus fréquemment, la proposition en comme quoi est accolée à un syntagme nominal, comme on le voit en (2a). En revanche, la percontative se trouve rarement dans cette fonction :

(31) Cette incertitude s'il a réussi son examen est pénible

En outre, la structure en comme quoi apparaît très souvent en début d'énoncé. Dans cette dernière configuration, elle rappelle les percontatives qui composent des titres :

(32) Comment une vieille prit soin de Candide, et comment il retrouva ce qu'il aimait. (Voltaire, Candide)

Elle a pu, pareillement composer un titre :

(33) Le titre de la première partie de Gribouille serait: Comment Gribouille se jeta dans la rivière par crainte d'être mouillé. Et la seconde : Comme quoi Gribouille se jeta dans le feu par crainte d'être brûlé.

Voyez si cela vous plaît. Bonsoir, portez-vous avant tout. (Sand, Correspondance)

mais ce n'est plus le cas aujourd'hui où elle survient insérée dans une suite discursive. Employé sans dépendre d'un verbe ni d'un nom, l'énoncé en comme quoi semble avoir perdu en grande partie sa valeur subordonnante. C'est pourquoi une virgule peut apparaître après ce groupe :

(34) a. "[...] Il faut dire ce qui a eu lieu, et a toujours lieu. Moi, on m'a toujours tenu la main. Ma vie a été un cadeau extraordinaire. Je suis touchée que le destin m'ait apporté cette "Dernière Lettre". Comme quoi, nous sommes tous reliés les uns aux autres" (Le Nouvel Observateur, 23-29 mai 2002)

Et il peut se paraphraser sans difficulté par un adverbe qui ne comporte aucune valeur subordonnante (cf. troisième partie) :

(3) g. Finalement, un journal ne se fait pas en un jour

Cependant il existe des contraintes dans l'emploi de comme quoi qui rappellent sa valeur subordonnante. Ce groupe ne peut pas se déplacer au sein de l'énoncé qu'il introduit :

(3) h. *Un journal comme quoi ne se fait pas en un jour.

(3) i. Un journal finalement ne se fait pas en un jour.

Ou alors il faudrait imaginer une pause significative :

(3) j. C'est un fait que l'épanouissement de ce journal, dans sa continuité a été exceptionnel. Pour cela il aura fallu 40 ans. Un journal (Comme quoi, hein...) ne se fait pas en un jour

Comme quoi formerait alors un nouvel énoncé, inachevé. En outre, il est difficile d'imaginer un ordre différent que celui trouvé dans les énoncés attestés : sujet - verbe - complément :

(3) k. *Comme quoi un journal se fait-il en un jour?

(3) 1. Finalement un journal se fait-il en un jour? 
Cet éloignement de la proposition en comme quoi par rapport aux percontatives explique peutêtre pourquoi elle peut se rapprocher de la complétive ( $\mathrm{cf}$. première partie). Ce n'est pas surprenant puisque c'est déjà le cas avec l'interrogative en comment, par exemple en (35) :

(35) Je ne comprends pas comment tu épouses une femme qui a deux enfants (exemple tiré de Sandfeld $1977: 71)^{13}$

Mais la dissemblance demeure entre la complétive et la structure en comme quoi. C'est très net pour les énoncés où la complétive ou la proposition en comme quoi suit un syntagme nominal. Comme quoi est d'un emploi plus aisé, pourvu qu'un sème en rapport avec le dire puisse se dégager. Avec ce groupe, il existe une analogie entre ce que dit ou montre le nom en question et le fait rapporté ; le groupe comme quoi accolé à un syntagme nominal se rapproche, plutôt que d'une complétive, du groupe disant + complétive :

\section{(8) c. Il a jamais reçu les messages disant que mon père était tombé}

A présent, examinons plus précisément les emplois de cette structure.

\section{Les emplois de la proposition en comme quoi}

Dans les premières constructions, telles que (1a) et (2a), le type de verbes et de noms avec lesquels s'emploie comme quoi nous permet de formuler l'hypothèse selon laquelle comme quoi forme un introducteur du discours indirect. D'après le cadre théorique proposé dans J. AuthierRevuz 1992-1993, tout discours indirect peut être considéré comme « un acte d'énonciation $\mathrm{E}$, défini par un couple d'interlocuteurs L, R, une situation SIT » dans laquelle prend place « un événement particulier qui est l'acte d'énonciation e qui est l'objet du message $\mathrm{M}$ de $\mathrm{E}$; e est luimême défini par 1, r, sit » (J. Authier-Revuz 1993 : 10). Revenons sur l'exemple (1a) :

\section{(1) a. La cousine a écrit comme quoi c'était toi pour les pièces de cinq francs} dans la cagnotte. (Queffelec, Les Noces barbares)

le locuteur 1, constitué par la cousine, aurait écrit ce qui est précisé dans la proposition en comme quoi. Le locuteur $\mathrm{L}$ reconstruit le message donné par 1: «e discours indirect est pleinement parole de L disant ce que signifie une parole de 1 » (J. Authier-Revuz 1978 : 64). Le discours rapporté en comme quoi instaure un double parcours.

Le premier parcours, grâce au pronom indéfini d'origine percontative, quoi, met en évidence les multiples paraphrases auxquelles pourrait avoir recours le locuteur L pour retranscrire la parole de 1 (J. Authier-Revuz $1978: 64)$ :

"la cousine a écrit que c'était toi pour les pièces de cinq francs dans la cagnotte ou elle a écrit quelque chose d'approchant"

Du pronom percontatif quoi du $17^{\mathrm{e}}$ siècle, il reste la suspension du savoir ("elle a écrit telle chose ou telle chose") que l'on peut percevoir dans les paraphrases : comme quoi introduit une structure qui laisse ouverte la question sur ce qui est dit dans les conditions de validité de l'énoncé. Nous sommes maintenant en mesure d'expliquer la différence de renvoi de quoi entre les énoncés $(2 a)$ et $(2 \mathrm{c})$ :

${ }^{13}$ Cf. également W. Wartburg et P. Zumthor $1989: 78$. 
(2) a. L'information précipitée comme quoi ce pâtre congelé, et peut-être épileptique, aurait été castré selon un rite initiatique du néolithique [...] se révèle, pour finir, sans fondement. (Sollers, Le Secret)

(2) c. L'information précipitée selon quoi / laquelle ce pâtre congelé, et peutêtre épileptique, aurait été castré selon un rite initiatique du néolithique [...] se révèle, pour finir, sans fondement

En (2c) quoi correspond à l'information précipitée alors qu'en (2a) il renvoie aux multiples paraphrases possibles :

"l'information précipitée dit que ce pâtre congelé [...] aurait été castré selon un rite initiatique du néolithique ou elle dit quelque chose d'approchant"

Le deuxième parcours, grâce à l'adverbe énonciatif comme, concerne la validité énonciative de ce qu'aurait rapporté le locuteur 1 . Le locuteur $L$ peut ne pas adhérer au discours de 1 qui ne renvoie pas forcément à quelque chose de réel. L'exemple (1a) pourrait se gloser de la façon suivante :

"le locuteur 1, c'est-à-dire la cousine, dit ça, mais ce qu'elle a dit n'est peutêtre pas vrai".

Cette analyse nous permet de percevoir la différence entre la complétive et la structure en comme quoi. La complétive connaît cette retranscription paraphrastique et le décalage entre ce qui est dit et le fait mais, contrairement à la proposition en comme quoi, elle ne le met pas en avant explicitement dans un double parcours.

Ces parcours ne s'appliquent pas de la même façon pour les trois types de construction. En effet, de (1a) à (3a), les paramètres propres au discours indirect disparaissent progressivement. Ils se manifestent avec le plus d'évidence lorsque tous les paramètres de la construction indirecte sont présents. C'est le cas lorsque la proposition en comme quoi constitue un complément essentiel d'un verbe déclaratif. Grâce au connecteur comme quoi, le locuteur L met en avant son absence de responsabilité devant les paroles du locuteur 1. Par la mise en place du double parcours, la proposition en comme quoi exige un écart possible entre le fait et le discours. C'est pourquoi, cette construction connaît des contraintes d'emploi. Elle est impossible avec les verbes qui n'acceptent aucune marge entre le fait et le discours sur ce fait, contrairement à la complétive :

(36) a. *Il sait comme quoi il a raté son entretien.

(36) b. Il sait qu'il a raté son entretien.

Avec les verbes qui cherchent à amoindrir la distance entre le fait et le discours, les énoncés en comme quoi refusent certains indices déictiques, comme ceux du pronom de la première personne ou du présent de l'indicatif, :

(37) *J'assure/*Je jure /* Je certifie comme quoi il a réussi son entretien.

Dans ce cas, le renvoi de $\mathrm{L}$ et 1 au même locuteur invalide toute possibilité d'écart entre le fait réel et le discours.

Lorsque la proposition en comme quoi est accolée à un nom, le discours indirect se présente avec moins de netteté. Les noms certes sont exclusivement en rapport avec le dire mais il n'est précisé que rarement de quel locuteur 1 il s'agit : 
(38) Il a jamais reçu les messages comme quoi mon père était tombé (énoncé entendu, 3 décembre 2001)

Le double parcours a moins d'incidences dans cette construction. Elle peut comporter des noms dont le sémantisme rappelle le flou lié à comme quoi, tels que bruits ou échos :

(39) Il y a eu des bruits qui ont couru comme quoi le Mollah Ohmar est allé au Pakistan (énoncé entendu, 13 novembre 2001, journal de 20 heures, France 2)

(40) J'avais eu des échos comme quoi le second allait se désister. (énoncé entendu, 2 décembre 2001)

Mais elle comprend sans aucun problème des noms qui refusent une distance entre le fait et ce qui est dit sur le fait, contrairement à la structure verbale :

(41) a. Il faut que je lui demande une attestation comme quoi je suis arrivée première (énoncé entendu, 30 juin 2002)

(41) b. ? Il atteste comme quoi je suis arrivée première

Les syntagmes nominaux sont le plus souvent précédés d'un déterminant indéfini. Mais le déterminant défini semble possible :

(41) c. Il faut que je lui demande l'attestation comme quoi je suis arrivée première

Dans ce type de construction, la distance entre le discours et le fait relaté propre au discours rapporté est moins ressentie. Comme alors servirait davantage à mettre en avant la conformité (M. Pierrard 1998) entre le discours et le fait raconté, de telle sorte qu'on en viendrait à oublier qu'il s'agit d'un discours rapporté.

Cette absence de distance entre le discours et le fait rapporté apparaît manifeste avec la dernière construction, lorsque comme quoi apparaît en début d'énoncé. Ni le locuteur 1 ni le support du discours ne sont indiqués : aucun signe du discours rapporté n'est manifeste. On pourrait penser que le locuteur 1 se construit par rapport aux phrases précédentes :

"comme les phrases précédentes le disent / le montrent, un journal ne se fait pas en un jour, ni en un tour de table"

Or, comme le locuteur L est généralement l'émetteur des phrases précédentes, c'est lui qui en fait prend en charge ce discours. Le locuteur 1 et le locuteur L ne constituent plus qu'un seul locuteur. Le locuteur L retranscrit la parole de 1 qui correspond en fait à son propre discours :

"comme je viens de le dire / de le montrer en évoquant ce fait particulier, un journal ne se fait pas en un jour, ni en un tour de table"

L'absence de tout signe manifeste du discours indirect réduit considérablement la portée de celui-ci. La marge entre le discours et le fait devient quasiment nulle : l'élément comme met en avant la conformité entre ce qui est dit et le fait.

Dans ce dernier type de construction, le groupe comme quoi connaît un sens discursif particulier dans l'organisation du discours. Il s'oppose à une idée généralement admise : 
(42) On est allés se promener alors qu'il pleuvait. Comme quoi on peut se promener lorsqu'il pleut.

En revanche, l'énoncé suivant :

(43) *Il fait beau, on est allés se promener. Comme quoi on peut se promener lorsqu'il fait beau

n'accepte pas le connecteur comme quoi parce qu'il est naturel de se promener lorsqu'il fait beau. Comme quoi réoriente un implicite généralement accepté ou donné comme évident ("lorsqu'il pleut, on ne se promène pas"). Il met en place la confrontation de deux points de vue, celui énoncé après comme quoi et cet implicite qui sera rejeté.

Il se rapproche de deux types de connecteurs. En premier lieu, il peut se paraphraser par des reformulatifs tels que finalement, somme toute etc. L'exemple (34a):

(34) a. "[...] Il faut dire ce qui a eu lieu, et a toujours lieu. Moi, on m'a toujours tenu la main. Ma vie a été un cadeau extraordinaire. Je suis touchée que le destin m'ait apporté cette "Dernière Lettre". Comme quoi, nous sommes tous reliés les uns aux autres" (Le Nouvel Observateur, 23-29 mai 2002)

pourrait se paraphraser par :

(34) b. Somme toute/finalement/tout bien considéré, nous sommes tous reliés les uns aux autres

Ce sont des connecteurs reformulatifs qui permettent d'établir un réexamen : « le locuteur indique qu'avant d'énoncer une nouvelle formulation, il a passé en revue tous les éléments d'un point de vue exprimé explicitement ou non ». (C. Rossari 1997 : 20) Une différenciation est établie, dans C. Rossari 1997 par exemple, entre les reformulatifs paraphrastiques (c'est-àdire, autrement dit) et les reformulatifs non paraphrastiques (somme toute, finalement). On pourrait se demander si comme quoi correspond à un reformulatif paraphrastique dans la mesure où il possède une teneur discursive et où il peut présenter, grâce à comme, un rapport sémantique entre ce que dit l'énoncé précédent et ce qui suit comme quoi :

(34) c. Comme les phrases précédentes le disent, nous sommes tous reliés les uns aux autres

Mais, entre ce que les phrases précédentes disent effectivement («Ma vie a été un cadeau extraordinaire. Je suis touchée que le destin m'ait apporté cette "Dernière Lettre" ») et ce qui est dit après comme quoi, "nous sommes tous reliés les uns aux autres », généralement, il est difficile de percevoir une équivalence sémantique entre ces phrases. On ne peut pas remplacer comme quoi par un des connecteurs paraphrastiques proposés dans E. Gülich et T. Kotschi 1983 ou dans C. Rossari 1997 :

(34) d. "Il faut dire ce qui a eu lieu, et a toujours lieu. Moi, on m'a toujours tenu la main. Ma vie a été un cadeau extraordinaire. Je suis touchée que le destin m'ait apporté cette "Dernière Lettre". *Autrement dit/ ${ }^{*} C$ 'est-àdire ${ }^{*}$ En d'autres termes, nous sommes tous reliés les uns aux autres ». 
En fait, un glissement de sens apparaît ; la reformulation ne s'établit pas entre ce qui suit comme quoi et ce que les phrases précédentes disent mais entre ce qui suit comme quoi et ce que les phrases précédentes impliquent ou montrent :

\section{(34) e. Comme les phrases précédentes le montrent, nous sommes tous reliés les uns aux autres.}

Le fait que comme quoi porte sur des implications corrobore l'idée que ce groupe ne reprend pas la proposition précédente telle quelle, mais tire de celle-ci des inférences (cf. première partie en 1.3.).

Dans ce cas, il ne s'agit plus d'une reformulation paraphrastique puisque le sens développé après comme quoi s'avère sensiblement différent de celui des énoncés précédents. C'est pourquoi le groupe comme quoi se rapproche davantage des connecteurs reformulatifs non paraphrastiques. Comme eux, il se trouve à l'origine de «la subordination rétroactive d'un mouvement discursif, éventuellement implicite, à une nouvelle intervention principale, du fait d'un changement de perspective énonciative » (E. Roulet $1987: 111)$.

En second lieu, de même que les reformulatifs non paraphrastiques, il se rapproche des connecteurs conclusifs. En effet, il a un fonctionnement "rétroactif». Il indique « un renvoi nécessaire à des constituants [...] cotextuellement rattachés ». (M. Schelling, 1982 : 66). Il « indique formellement un acte directeur» (67). En outre, « du point de vue de [ses] instructions argumentatives, il opère après-coup une coorientation des arguments sur lesquels il porte »(67). En cela, il ressemble aux reformulatifs et conclusifs que sont par exemple finalement, en somme. Mais il se différencie d'eux par son instruction argumentative propre : il opère une conformité entre ce que montrent les phrases précédentes et ce qui est énoncé après lui. Et ce que montrent les phrases précédentes s'oppose à une idée reçue ou considérée comme admise, par exemple en (3a) : "un journal se fait rapidement". L'énoncé précédent constitue une preuve, un argument, pour opérer un commentaire. Parfois le locuteur opère ce commentaire en gardant les mêmes instances d'énonciation, comme en (34a). Le temps employé peut signifier le résultat, l'aspect accompli (extensif) pour le passé composé :

(44) Dès 1975, les journaux relataient ses menaces de mort contre un autre journaliste [...]. Comme quoi le personnage n'a pas dérapé en 1979 : il n'avait tout simplement pas changé. (TéléObs, 7-13 mars 2002)

Le plus fréquemment, le groupe comme quoi apporte une généralisation. Sont utilisées alors des marques de généralisation, présent gnomique, verbes impersonnels, pronoms indéfinis :

(45) Le petit monde de Wiaz, c'est le vaste monde du pouvoir, de la guerre et du malheur. Il se hâte d'en rire... Comme quoi on peut avoir de l'esprit et et du coeur. Wiaz expose les deux à la Scam jusqu'au 26 avril. (Le Nouvel Observateur, 4-10 avril 2002)

(46) Si léger et pourtant si complet! Avec seulement $85 \mathrm{~g}$, le Sagem MY1078 vous fera vibrer, jouer, surfer et communiquer aux sons des nouvelles technologies! Comme quoi, on peut être à la fois un poids plume et un poids lourd de fonctionnalités... (Publicité pour un téléphone portable)

Ces marques de généralisation conviennent également à l'apport conclusif de comme quoi. En effet, elles rapprochent la structure en comme quoi d'une morale. C'est pourquoi, les énoncés en comme quoi occupent parfois des emplacements stratégiques du texte, la fin d'un 
paragraphe ou même d'un article, ainsi en (34a). Le groupe comme quoi peut également apparaître seul :

(47) - A trente ans, ce seront des loques

- Pourtant ils [coureurs cyclistes du tour de France] ont l'air en bonne santé, minces, musclés... Comme quoi (énoncé entendu, 29 juin 2002)

L'énoncé inachevé en comme quoi se comprend alors grâce au contexte :

"comme quoi, le sport, c'est peut-être pas une panacée"

Ce commentaire inachevé du locuteur s'explicite facilement puisque comme quoi fonctionne sur le même schéma : il suffit de voir ce que peuvent impliquer les phrases précédentes et à quelle idée reçue l'énoncé en comme quoi peut s'opposer.

\section{Conclusion}

Ainsi la proposition en comme quoi provient d'une structure interrogative. Elle se distingue de la relative, de la complétive et de l' intégrative ; quoi ne forme ni un relatif ni un intégratif. De nos jours, la proposition en comme quoi s'avère difficile à catégoriser puisqu'elle présente de nombreuses différences avec les interrogatives. Le groupe comme quoi apparaît comme un connecteur énonciatif. Il introduit le discours indirect en présentant explicitement un double parcours, l'un sur les conditions d'énonciation entre le discours qu'aurait prononcé le locuteur et les faits, l'autre sur les paraphrases émises pour rapporter le discours de ce locuteur. Mais le discours indirect est plus ou moins évident, selon les structures en comme quoi. C'est pourquoi, il peut manifester un écart entre les deux locuteurs (construction à partir du verbe) ou au contraire fonctionner comme un signe d'adhésion (structure en comme quoi en début d'énoncé). Dans ce dernier cas, il apparaît comme un opérateur de reformulation.

\section{Summary}

The aim of this paper is to explain how the group comme quoi works. The subordinate clause with comme quoi differs from a relative clause, a completive clause or an integrative clause. Considering its use in indirect speech (verb + comme quoi / nominal group + comme quoi), it seems that this group introduces an indirect interrogative (percontative), with quoi as interrogative (percontative) pronoun and comme as an integrative adverb concerning enunciation. Nevertheless, we show the differences between the clause with comme quoi and the percontative clause. Actually, being first an interrogative group about the manner (in the seventeenth century), it became a word introducing an indirect speech with an enunciative meaning.

Florence Lefeuvre

8 rue de Reims

75013 Paris

\section{REFERENCES}

Authier-Revuz, Jacqueline. 1993. Repères dans le champ du discours rapporté. L'information grammaticale. 56. p. 10-15. 
Authier-Revuz, Jacqueline. 1992. Repères dans le champ du discours rapporté. L'information grammaticale. 55. p. 38-42.

Authier-Revuz, Jacqueline. 1978. Les formes du discours rapporté. Remarques syntaxiques et sémantiques à parir des traitements proposés. DRLAV. 17. p. 1-87.

Béchade Hervé-D, 1994. Grammaire française. Paris : PUF.

Chiflet, Laurent (Père -). 1659. Essay d'une parfaite Grammaire de la langue française. Anvers, Jacques Van Meurs (rééd Genève, Slatkine reprints, 1973).

Combettes, Bernard. 1989. Discours rapporté et énonciation: trois approches différentes. Pratiques. 64. p. 111-122.

Damourette, Jacques; Pichon, Édouard. 1911-1940. Des mots à la pensée. Paris : Editions d'Artrey.

Fournier, Nathalie. 1998. Grammaire du Français classique. Paris : Belin.

Grevisse, Maurice. 1988. Le Bon Usage. Gembloux (Belgique) : Duculot.

Gülich, Elizabeth ; Kotschi, Thomas, 1983 : Les marqueurs de la reformulation paraphrastique. Cahiers de linguistique française. 5, 305-351.

Le Bidois Georges ; Le Bidois Robert. 1971 : Syntaxe du Français moderne, Paris : Editions A. et J.Picard.

Lefeuvre, Florence. 2001. La grammaticalisation du pronom indéfini quoi. Travaux linguistiques du Cerlico. 14. p. 181-202.

Lefeuvre, Florence. 2002. Comme quoi. Traits d'Union. Caen : Presses Universitaires de Caen. p. 73-85.

Lefeuvre, Florence (à paraître a). Comme quoi en diachronie. Verbum.

Lefeuvre, Florence (à paraître b). Le groupe préposition + quoi en début d'énoncé, Actes $d u$ colloque "Littérature et Linguistique " (Chambéry, novembre 2002).

Lefeuvre Florence (à paraître c). Le pronom relatif quoi, Actes du colloque "Subordination et coordination» (Brest, novembre 2003).

Lefeuvre Florence (à paraître d). Le pronom quoi renvoie-t-il à de l'inanimé, Le Français moderne.

Le Goffic, Pierre. 1994. Indéfinis, interrogatifs, relatifs : parcours avec ou sans issue. Faits de Langue. 4. p. 31-40.

Le Goffic, Pierre. 1993. Grammaire de la phrase française. Paris : Hachette.

Le Goffic, Pierre. 1991. Comme, adverbe connecteur intégratif : éléments pour une description, Travaux linguistiques du Cerlico. ${ }^{\circ} 4$. p. 11-31.

Mirault, Hugues. 1997: La Syntaxe des propositions relatives, Etude contrastive des différences structurelles d'ordre syntaxique entre le français et l'allemand. Paris, Peter Lang.

Muller, Claude. 1996. La Subordination en français. Paris : Colin.

Pierrard, Michel. 1998. Comme « relatif à antécédent» en ancien français : grammaticalisation de la proforme indéfinie. Travaux de linguistique. 36. p. 127-146.

Riegel, Martin; Pellat, Jean-Christophe; Rioul, René. 1994. Grammaire méthodique du français. Paris : PUF.

Rosier, Laurence. 1999. Le Discours rapporté. Bern : Peter Lang.

Rossari, Corinne. 1997. Les opérateurs de reformulation. Bern : Peter Lang.

Roulet, Eddy et al. 1985. L'articulation du discours en français contemporain. Bern : Peter Lang.

Roulet, Eddy. 1987. Complétude interactive et connecteurs reformulatifs. Cahiers de linguistique française. 8. 111-140.

Sandfeld K. 1977. Syntaxe du français contemporain, Les propositions subordonnées. Genève. Droz.

Schelling, Marianne. 1982. Quelques modalités de clôture, les conclusifs : finalement, en somme, au fond, de toute façon. Cahiers de linguistique française. 4. p. 63-106. 
Vaugelas Favre (de), Claude. 1647. Remarques sur la Langue française.Paris, Vve Camusat et Le Petit (rééd. de Stricher, Paris, Droz, 1934).

Von Wartburg, Walter; Zumthor, Paul. 1947 (4e éd. 1989). Précis de syntaxe du français contemporain. Ed. Francke. Bern.

Wilmet, Marc. 1997. Grammaire critique du français. Paris : Hachette. 